\title{
A generic constitutive model for the passive porcine coronary artery
}

\author{
Chantal N. van den Broek • Arjen van der Horst • \\ Marcel C. M. Rutten - Frans N. van de Vosse
}

Received: 1 December 2009 / Accepted: 20 May 2010 / Published online: 17 June 2010

(C) The Author(s) 2010. This article is published with open access at Springerlink.com

\begin{abstract}
Constitutive models describing the arterial mechanical behavior are important in the development of catheterization products, to be used in arteries with a specific radius. To prove the possible existence of a constitutive model that, provided with a generic set of material and geometric parameters, is able to predict the radiusspecific mechanical behavior of a coronary artery, the passive pressure-inner radius $\left(P-r_{i}\right)$ and pressure-axial force change $\left(P-\Delta F_{z}\right)$ relations of seven porcine left anterior descending coronary arteries were measured in an in-vitro set-up and fitted with the model of Driessen et al. in J Biomech Eng 127(3):494-503 (2005), Biomech Model Mechanobiol 7(2):93-103 (2008). Additionally, the collagen volume fraction, physiological axial pre-stretch, and wall thickness to inner radius ratio at physiological loading were determined for each artery. From this, two generic parameter sets, each comprising four material and three geometric parameters, were obtained. These generic sets were used to compute the deformation of each tested artery using a single radius measurement at physiological loading as an arteryspecific input. Artery-specific $P-r_{i}$ and $P-\Delta F_{z}$ relations were predicted with an accuracy of $32 \mu \mathrm{m}(2.3 \%)$ and $6 \mathrm{mN}$ (29\% relative to $\Delta F_{z}$-range) on average compared to the relations measured in-vitro. It was concluded that the constitutive model provided with the generic parameters found in this study can well predict artery-specific mechanical behavior.
\end{abstract}

C. N. van den Broek $(\bowtie) \cdot$ A. van der Horst · M. C. M. Rutten

F. N. van de Vosse

Department of Biomedical Engineering,

Eindhoven University of Technology, PO Box 513,

5600 MB Eindhoven, The Netherlands

e-mail: C.N.v.d.Broek@tue.nl
Keywords Coronary - Parameters - Model - General set · Generic description

\section{Introduction}

The percutaneous transluminal coronary angioplasty procedure is one of the most commonly used methods to restore blood flow in case of coronary artery disease. Although this procedure has proven to be very successful, restenosis may still occur. Therefore, in recent years, much attention has been paid to the development of drugeluting stents with the aim to reduce the risk of restenosis. Apart from pharmacological developments, mechanical developments in the treatment of coronary artery disease are also of major importance. The development of catheters with such mechanical properties that they can be introduced smoothly into the arterial system without damaging the arterial wall and its endothelial inner layer too much is therefore of great interest. Also, less damage of the endothelium and other layers of the arterial wall as a result of the inflation of the balloon at the location of the stenosis might decrease the restenosis rate. Therefore, a better understanding of the mechanical interaction of catheterization products and the arterial wall may be helpful in the improvement of those products. For this, a generic constitutive model, describing the coronary arterial wall behavior under load, is needed.

Several experimental methods have been developed to characterize arterial wall mechanics, e.g. uniaxial and biaxial tensile tests with arterial rings and strips (Cox 1983; Lally et al. 2004; Holzapfel et al. 2005), and inflation and axial extension tests with longer arterial segments ( $\mathrm{Lu}$ et al. 2004). The latter is more preferable as the tubular shape of the artery is preserved (Hayashi 1993; 
Humphrey and $\mathrm{Na}$ 2002). In addition, the axial force needed to keep the imposed axial pre-stretch during inflation constant can be measured simultaneously. The thus measured pressure-radius and pressure-axial force relations can then be fitted with the use of a proposed constitutive model.

A commonly used model is the one developed by Holzapfel and Gasser (2000) in which the arterial wall is described as a fiber-reinforced material, with the fibers representing the collagen. This model was adapted by Driessen et al. (2005, 2008), incorporating a distribution on the fiber orientation. To obtain a full mechanical characterization of the arterial tissue, the reference state needs to be known. Therefore, the pressure needs to be varied below the physiological range, and the axial pre-stretch needs to be known. The disadvantage, however, is that such measurements can only be conducted in an in-vitro environment.

It would be of great value when the arterial pressureradius and pressure-axial force relation could be predicted over a larger pressure range with a single in vivo radius measurement at physiological pressure and axial pre-stretch. The objective of this study is therefore to prove the possible existence of a constitutive model that, provided with a generic set of material and geometric parameters, is able to predict the radius-specific mechanical behavior of a coronary artery. To achieve this goal, the arterial wall behavior, including pressure-radius and pressure-axial force measurements, was determined for seven porcine left anterior descending coronary arteries (LADs). The LAD was used as this coronary is relatively straight and hardly tapering (about $5 \% / \mathrm{cm}$ ). The measurements were fitted with the constitutive model of Driessen et al. $(2005,2008)$. It is expected that the physiological incorporated fiber distribution in this model will improve the fit and will therefore lead to a better generic description of the artery. From those fits, two different generic parameter sets $\left(\bar{\Upsilon}\right.$ and $\Upsilon_{m}$ ), each comprising four material parameters, were obtained. The first, by taking the mean of the parameters derived for each $\mathrm{LAD}$, and the second, by fitting the parameters to the mean behavior of all LADs, so combining all measurements. Moreover, the wall thickness to inner radius ratio, the physiological axial pre-stretch, and the collagen fiber fraction were determined for the seven arteries and averaged. Next, the generic parameter sets and the averaged geometric parameters, together with the radius determined at physiological pressure of a particular artery, were used as an input for the model to predict the arterial wall behavior of that artery. The thus determined pressure-inner radius and pressure-axial force relations of each artery were validated by comparing with the experimentally measured relations of each LAD.

\section{Materials and methods}

\subsection{Sample preparation}

Porcine hearts $(n=7)$ were obtained at a local slaughterhouse. Immediately after exsanguination of the animal, the heart was removed and immersed in a cold cardioplegia solution to stop its activity. Hearts were transported to the laboratory on ice. Within $3 \mathrm{~h}$ after explantation, segments of the LAD, 20-30 $\mathrm{mm}$ in length, were excised from the heart. Side branches were closed using arterial clips, and loose connective tissue was removed. Two cannulae were inserted at the proximal and distal end of each segment and fixed with a suture. The ex vivo, unstretched segment length $\left(l_{0}\right)$ was determined by measuring the length between the two sutures. As the contraction state of the heart after exsanguination is unknown, the pre-stretched segment length on the heart was not used.

\subsection{Experimental set-up}

The arterial segment was placed in an in-vitro set-up (Fig. 1) and mounted at $l_{0}$ between two stainless steel tubes immersed in a Kreb's solution. The cannulae were connected to silicone rubber tubes using polypropylene connector parts, making up a closed circuit together with the organ bath. Part of the circuit consisted of a pressure pump, which was driven with a proportional pneumatic valve (Festo, The Netherlands), inducing a pulsatile pressure. The pressure was measured by a pressure transducer (P10EZ, BD, USA). The Kreb's solution was pumped through a silicone tube that was immersed in a warm water bath heating up the Kreb's solution. The heated solution was then returned to the organ bath. A temperature sensor in the organ bath allowed temperature control of the fluid surrounding the arterial segment, keeping it at $38^{\circ} \mathrm{C}$.

The tube at the proximal end of the segment was fixed to a stainless steel rod perpendicular to the tube. The rod was fixed to a linear actuator and a controller (235.5 DG and C843, Physik Instrumente, Germany) by which the pre-stretched length of the segment could be controlled. The distal cannula was connected to an axial force transducer via a rigid axial bearing, measuring the reduced axial force $\left(F_{z}\right.$, Eq. 8$)$ during the inflation experiment. Changes in arterial inner diameter were recorded using an ultrasound scanner with a linear probe ( $8 \mathrm{MHz}$, Esaote Europe, The Netherlands) combined with an arterial analyzer (Art.Lab, Esaote Europe, The Netherlands). The ultrasound probe was placed in a 3D manipulator for easy positioning. The inner diameter $\left(D_{i}\right)$ was measured along 32 lines/cm in B-Mode (30 frames/s). The pressure $(P)$ signal was recorded simultaneously with $D_{i}$. The proportional valve controlling the pressure, the linear actuator, and $P-F_{z}$ data 
Fig. 1 In-vitro set-up for the arterial mechanical behavior assessment

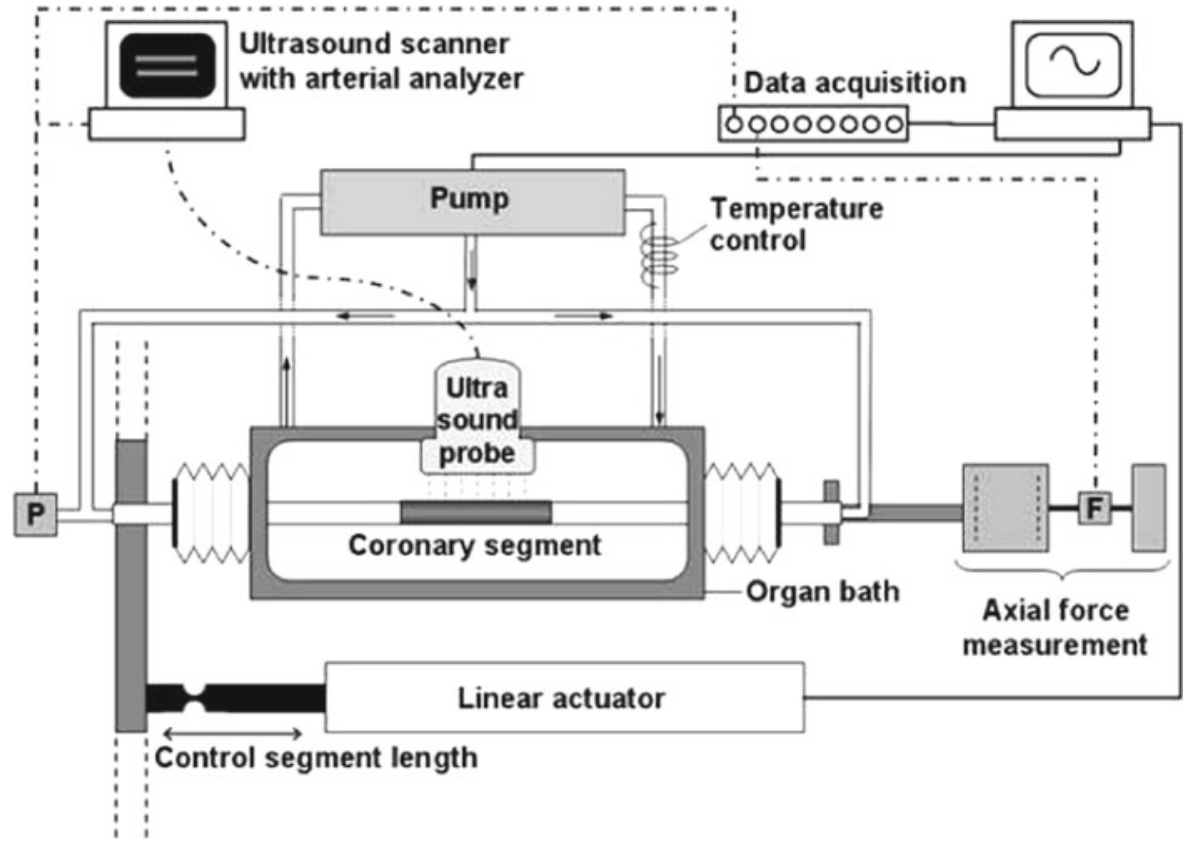

acquisition were controlled with Labview software (National Instruments, USA).

\subsection{Test protocol}

Arterial segments were allowed to equilibrate at $l_{0}$ in a Kreb's solution at $38^{\circ} \mathrm{C}$ for $30 \mathrm{~min}$. Subsequently, papaverine $\left(10^{-4} \mathrm{M}\right)$ was added inducing arterial relaxation and a moderate flow through the segment was induced. After $15 \mathrm{~min}$, flow was stopped and a cyclic pressure load at a frequency of $1 \mathrm{~Hz}$ was applied. Transmural pressure varied from $0 \mathrm{kPa}$ to the maximum pressure that could be achieved without inducing arterial buckling, with a maximum of $16 \mathrm{kPa}$. The artery was stretched axially at a strain rate of $0.01 / \mathrm{s}$ until the axial force amplitude resulting from the cyclic pressure was minimized. This was defined as the physiological length of the segment ( $l_{\text {phys }}$; (Weizsäcker et al. 1983)), with the corresponding physiological axial pre-stretch being $\lambda_{z \text {,phys }}=l_{\text {phys }} / l_{0}$. The arterial segment was axially preconditioned by stretching the artery from $l_{0}$ to $l_{\text {phys }} 5$ times at a strain rate of $0.01 / \mathrm{s}$, after which the axial force response did not change anymore.

Next, the segment was cyclically pressurized from 0-16 kPa with a sinusoidal function $(1 \mathrm{~Hz})$ at $l_{\text {phys }}$. The $P$-inner radius $\left(P-r_{i}\right)$ and $P-F_{z}$ relations were measured for one pressure cycle, once the relations were reproducible. The pressure signals from the ultrasound system and the data acquisition system were used to synchronize the $P-r_{i}$ and $P-F_{z}$ recordings. Next, the change in axial force during a pressure cycle, $\Delta F_{z}$, was obtained by subtracting the axial force at $P=0$ from the axial force data. As some hysteresis was present, the $P-r_{i}$ and $P-\Delta F_{z}$ relations at increasing pressure load were averaged with the corresponding relations at decreasing pressure load. The resulting $P-r_{i}$ and $P-\Delta F_{z}$ relations were used to fit with the model (see Sect. 2.6).

\subsection{Morphologic analysis}

Histological analysis was performed to investigate the morphology of the arterial wall. At the end of the test protocol, a small ring was cut from the middle part of each arterial segment and fixed in a $10 \%$ formalin solution in PBS for 12-24h. Next, segments were embedded in paraffin. From each segment, $6 \mu \mathrm{m}$ thick circular tissue sections were cut and placed on poly-L-lysine coated glass slides. After rehydration of the tissue sections, major structural components in the vascular tissue were visualized using a Masson Trichrome (MTC) staining, coloring muscular and connective tissue pink and collagen blue. Pictures of the tissue sections were taken at a $10 \times$ magnification. An estimate of the average cross-sectional area of the unloaded segment $\left(A_{0}\right)$, to be used in Sect. 2.6, was determined by measuring the inner and outer circumference $\left(C_{i}\right.$ and $C_{o}$ resp.) of the tissue section $\left(A_{0}=\left(C_{o}^{2}-C_{i}^{2}\right) / 4 \pi\right)$. The relative thickness of the adventitia layer, used as a measure of the collagen fiber volume fraction in the arterial wall $\left(\phi_{f}\right)$, was determined by measuring the inner circumference of the adventitia layer $\left(C_{a}\right)$ $\left(\phi_{f}=\left(C_{o}-C_{a}\right) /\left(C_{o}-C_{i}\right)\right)$. This approximation for the collagen fiber fraction is motivated by the fact that the main component of the adventitia is collagen and the media mainly consists of smooth muscle cells (Rhodin 1980). It is assumed that the overestimation of collagen in the adventitia is mostly compensated by neglecting the collagen in the media. 


\subsection{Constitutive model}

To describe the arterial mechanical response to axial extension and intraluminal pressure variations, the artery is considered an incompressible (Chuong and Fung 1984), thick-walled tube. The Cauchy stress $(\sigma)$ in the arterial wall is calculated using the constitutive model of Driessen et al. (2005, 2008). In this model, the arterial wall is described as a fiber-reinforced material with a spatial distribution of the fiber orientation, the fibers representing the collagen. Using the rule of mixtures in the direction of the fibers, the general constitutive equation is given by

$\sigma=-p \mathbf{I}+\hat{\tau}+\sum_{i=1}^{N} \phi_{f}^{i}\left(\tau_{f}^{i}-\mathbf{e}_{f}^{i} \cdot \hat{\tau} \cdot \mathbf{e}_{f}^{i}\right) \mathbf{e}_{f}^{i} \otimes \mathbf{e}_{f}^{i}$,

with $p$ the hydrostatic pressure, $\hat{\tau}$ the isotropic matrix stress, and $\tau_{f}^{i}, \phi_{f}^{i}$, and $\mathbf{e}_{f}^{i}$ the stress, relative volume fraction and current orientation of fiber $i$, respectively, and $N$ the number of fiber families. For the isotropic part of the stress, a Neo-Hookean description with a shear modulus $G$ is used:

$\hat{\tau}=G(\mathbf{B}-\mathbf{I})$,

with the left Cauchy-Green deformation tensor (B) given by $\mathbf{B}=\mathbf{F} \cdot \mathbf{F}^{T}$, with $\mathbf{F}$ the deformation gradient tensor. To describe the non-linear stress-strain behavior of arteries, the stress of fiber $i$ is described with an exponential relation:

$\tau_{f}^{i}=2 k_{1} \lambda_{f}^{i^{2}}\left(\lambda_{f}^{i^{2}}-1\right) e^{k_{2}\left(\lambda_{f}^{i^{2}}-1\right)^{2}}$,

where $\lambda_{f}^{i}$ is the stretch of fiber $i$ and $k_{1}$ and $k_{2}$ are a stiffness and dimensionless fiber parameter. The direction of fiber $i$ in the deformed configuration is derived from the fiber direction in the undeformed configuration $\left(\mathbf{e}_{f_{0}}^{i}\right)$, the fiber stretch $\left(\lambda_{f}^{i}\right)$ and $\mathbf{F}$ by

$\lambda_{f}^{i} \mathbf{e}_{f}^{i}=\mathbf{F} \cdot \mathbf{e}_{f_{0}}^{i}, \quad \lambda_{f}^{i}=\left\|\mathbf{F} \cdot \mathbf{e}_{f_{0}}^{i}\right\|=\sqrt{\mathbf{e}_{f_{0}}^{i} \cdot \mathbf{C} \cdot \mathbf{e}_{f_{0}}^{i}}$,

with $\mathbf{C}=\mathbf{F}^{T} \cdot \mathbf{F}$, the right Cauchy-Green deformation tensor and

$\mathbf{e}_{f_{0}}^{i}=\cos \left(\beta^{i}\right) \mathbf{e}_{\theta}+\sin \left(\beta^{i}\right) \mathbf{e}_{z}$,

with $\beta^{i}$ the angle of fiber $i$ relative to the circumferential direction in the unloaded configuration. The volume fraction of fiber $i$ is defined by a periodic approximation of the normal probability distribution (Driessen et al. 2008) as

$\phi_{f}^{i}\left(\beta^{i}\right)=A \exp \left[\frac{\cos \left(2\left(\beta^{i}-\beta\right)\right)+1}{\gamma}\right]$,

with $\beta$ and $\gamma$ the main fiber angle and the dispersion of the fiber volume fraction distribution, respectively, and $A$ a scaling factor to ensure the total volume fraction of fibers equals $\phi_{f}$.
The prescribed internal lumen pressure $P$ can be calculated using the boundary conditions $\left.\sigma_{r r}\right|_{r=r_{o}}=0$ and $\left.\sigma_{r r}\right|_{r=r_{i}}=-P$ by:

$P=\int_{r_{i}}^{r_{o}}\left(\sigma_{\theta \theta}-\sigma_{r r}\right) \frac{d r}{r}$

with $P$ the internal lumen pressure. The deformation resulting from a known load with internal pressure $P$ and axial extension $\lambda_{z}$ can then be found by solving (7) following the procedure described in e.g. Driessen et al. (2004). When the deformation is known, the reduced axial force $F_{z}$ needed to keep the axial stretch during inflation constant can be computed using:

$F_{z}=2 \pi \int_{r_{i}}^{r_{o}} \sigma_{z z} r d r-\pi r_{i}^{2} P$.

\subsection{Fit procedure}

The model (Sect. 2.5, with $N=9$ and $\gamma=10^{\circ}$ ) was fitted to the experimental data of each $\operatorname{LAD} j(j=\{1, \ldots, 7\})$ obtained in Sect. 2.3, by estimation of the unloaded inner radius $\left(R_{i}\right)$ and wall thickness $\left(H_{0}\right)$, and optimization of the four fit parameters $\left\{G, k_{1}, k_{2}, \beta\right\}$, using the algorithm as described in Fig. 2. First, an estimate of the unloaded geometry $\left(R_{i}^{(0)}\right.$ and $\left.H_{0}^{(0)}\right)$ based on $A_{0}$ (Sect. 2.4, $A_{0}=$

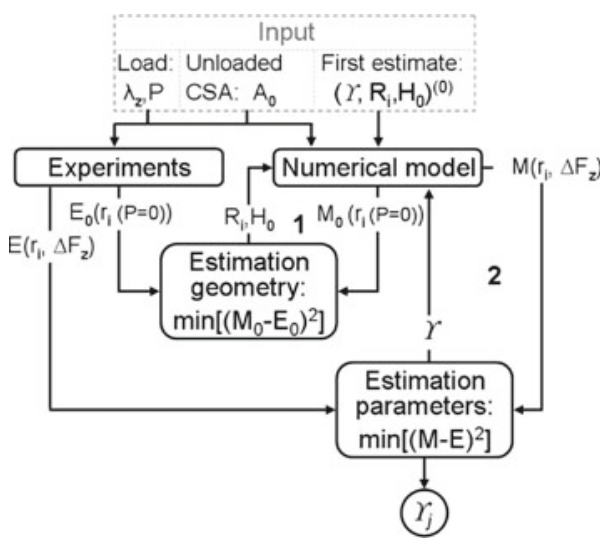

Fig. 2 A schematic representation of the protocol used to estimate the unloaded geometry and fit the model parameters to the experimental data, using the Levenberg-Marquardt method. A load $\left(\lambda_{z}, P\right)$, unloaded cross-sectional area (CSA) and estimate of the model parameters and unloaded geometry $\left(\left(\Upsilon, R_{i}, H_{0}\right)^{(0)}\right)$ were given as an initial input for the numerical model. First, the unloaded geometry was estimated by fitting $r_{i}(P=0)$ (loop 1). Next, the model parameters were estimated by fitting the experimental $r_{i}$ and $\Delta F_{z}$ data (loop 2), using the estimated unloaded geometry as an input. $\Upsilon_{j}$ is the final optimal parameter set of LAD $j . \mathbf{M}_{0}$ and $\mathbf{E}_{0}$ are the model and experimental output for $r_{i}$ $(P=0)$ and $\mathbf{M}$ and $\mathbf{E}$ are the model and experimental output for $r_{i}$ and $\Delta F_{z}(P=0-16 \mathrm{kPa})$ 
$\pi\left(\left(R_{i}^{(0)}+H_{0}^{(0)}\right)^{2}-\left(R_{i}^{(0)}\right)^{2}\right)$ of each data set $j(j=1-7)$ and the model parameters $\left(\Upsilon^{(0)}\right)$ were used as an input for the model for the first iteration. Next, $R_{i}$ and $H_{0}$ were optimized by minimization of the sum of squares of the deviation between the experimentally measured $r_{i}(P=0)\left(\mathbf{E}_{0}\right)$ and the model output for $r_{i}(P=0)\left(\mathbf{M}_{0}\right)$. Subsequently, the sum of squares of the deviations between the experimentally measured $r_{i}$ and $\Delta F_{z}(P=0-16 \mathrm{kPa})(\mathbf{E})$ and the model output for $r_{i}$ and $\Delta F_{z}$ for the same pressure range (M) was minimized using the Levenberg-Marquardt algorithm to optimize the four fit parameters. Each time the parameter set was changed, also during the Levenberg Marquardt process (loop 2, Fig. 2), a new estimate of the unloaded geometry was made. During the entire fit procedure, incompressibility was maintained by keeping the volume constant. For the estimation of the unloaded geometry this meant that the unloaded cross-sectional $A_{0}$ was kept constant as $\lambda_{z}$ is 1 for that case. In the loaded configuration at $\lambda_{z \text {,phys }}$ the loaded cross-sectional area, defined as $A_{\text {phys }}=A_{0} / \lambda_{z \text {,phys }}$, was kept constant to meet the incompressibility condition. The resulting individual parameter set of LAD $j$ is referred to as $\Upsilon_{j}=\left\{G_{j}, k_{1, j}, k_{2, j}, \beta_{j}\right\}$.

\subsection{Generic description of the porcine LAD}

To determine whether the estimated parameters of Sect. 2.6 can be used to obtain a generic description of the porcine LAD, two generic parameter sets were defined (Fig. 3). The first generic parameter set was obtained by averaging the seven parameter sets $\Upsilon_{j}$ (Sect. 2.6), giving the averaged parameter set $\bar{\Upsilon}\left(=\left\{\bar{G}, \bar{k}_{1}, \bar{k}_{2}, \bar{\beta}\right\}\right)$. To obtain the second generic parameter set, the experimental data of LAD 1-7 were averaged. This data set was fitted with the model using the fit procedure as described in Sect. 2.6, by which the generic parameter set $\Upsilon_{m}$ was obtained. The sets $\bar{\Upsilon}$ and $\Upsilon_{m}$ were used as an input for the model. Moreover, three

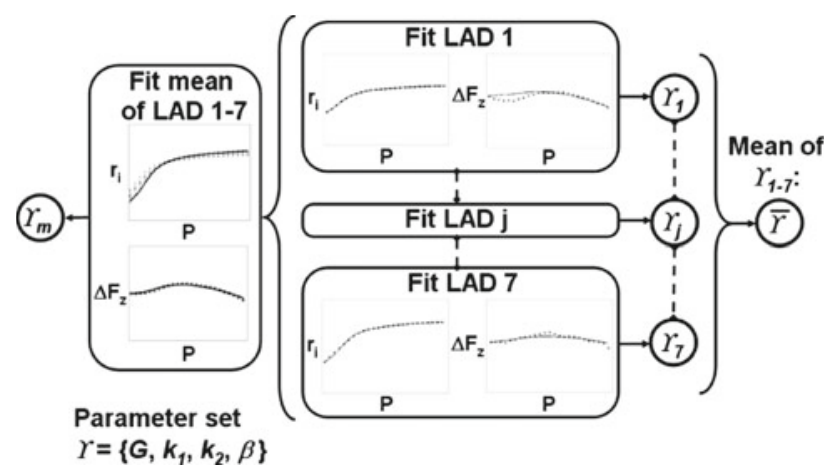

Fig. 3 The different obtained parameter sets, $\Upsilon_{j}$ being the parameter set fitted to the experimental data of LAD $j, \bar{\Upsilon}$ the averaged parameter set of the sets $\Upsilon_{1-7}$, and $\Upsilon_{m}$ the parameter set derived by fitting the averaged data of LAD 1-7 generic geometric parameters were obtained by averaging: $\phi_{f}, \lambda_{z}$,phys and the ratio $\kappa=h / r_{i}$ at physiological loading ( $P \approx 13.3 \mathrm{kPa}$ and $\lambda_{z}$,phys $)$ of the seven LADs considered resulting in $\bar{\phi}_{f}, \bar{\lambda}_{z \text {,phys }}$, and $\bar{\kappa}$ respectively. Both $\bar{\Upsilon}$ and $\Upsilon_{m}$, and $\bar{\phi}_{f}, \bar{\lambda}_{\text {phys }}$, and $\bar{\kappa}$ were used as an input for the model and applied to each LAD $j$. The experimentally measured $r_{i}$ at physiological mean pressure $(\approx 13.3 \mathrm{kPa})$ and physiological axial pre-stretch $\left(r_{i, \text { phys }}\right)$, was used as an LAD-specific input. The unloaded geometry (i.e. $R_{i}$ and $H_{0}$ ) was determined iteratively by fitting the model to the experimentally measured $r_{i, \text { phys }}$ and keeping the known cross-sectional area $\left(A_{0}=\bar{\lambda}_{z, \text { phys }} \pi\left(\bar{\kappa}^{2}+2 \bar{\kappa}\right) r_{i, \text { phys }}^{2}\right)$ constant.

\subsection{Data analysis}

The model approximations of the $P-r_{i}$ relations were analyzed by determining the mean relative deviations of the approximated $P-r_{i}$ relations with $\Upsilon_{j}, \bar{\Upsilon}$, or $\Upsilon_{m}$ from the experimentally derived relation for each $\operatorname{LAD} j$, i.e.

$\delta_{r}=\frac{1}{n_{k}} \sum_{k=1}^{n_{k}}\left|\frac{r_{i, M}(k)-r_{i, E}(k)}{r_{i, E}(k)}\right|$

with $n_{k}$ the number of datapoints, and $r_{i, M}(k)$ and $r_{i, E}(k)$ the model approximation and experimentally determined $r_{i}$ at data point $k$, respectively.

As a measure of the quality of the model approximation of the $P-\Delta F_{z}$ relations, the relative deviations of the approximated axial force amplitude from the experimentally measured amplitude were determined. This was done by determining the deviation of the model approximation of the maximum and minimum of the $\Delta F_{z}$ range from the experimentally measured maximum and minimum, relative to the experimentally measured $\Delta F_{z}$ range for each $\operatorname{LAD} j$, i.e.

$$
\begin{aligned}
\delta_{F}= & \frac{1}{2} \frac{\left|\min \left(\Delta F_{z, M}\right)-\min \left(\Delta F_{z, E}\right)\right|}{\max \left(\Delta F_{z, E}\right)-\min \left(\Delta F_{z, E}\right)} \\
& +\frac{1}{2} \frac{\left|\max \left(\Delta F_{z, M}\right)-\max \left(\Delta F_{z, E}\right)\right|}{\max \left(\Delta F_{z, E}\right)-\min \left(\Delta F_{z, E}\right)}
\end{aligned}
$$

with $\Delta F_{z, M}$ and $\Delta F_{z, E}$ the model approximation of and the experimentally determined $\Delta F_{z}$, respectively.

\section{Results}

Arterial geometry and mechanical behavior were determined for seven porcine LADs. Figure 4 shows an MTC-stained histological section from which the LAD-specific unloaded cross-sectional area was derived to be used for fitting ( $A_{0}$, Table 1). Moreover, the fiber fraction $\phi_{f}$ was derived from the thickness of the adventitia of the histology sections and was $0.41 \pm 0.05$ on average. The average physiological pre-

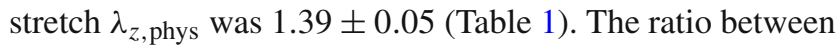




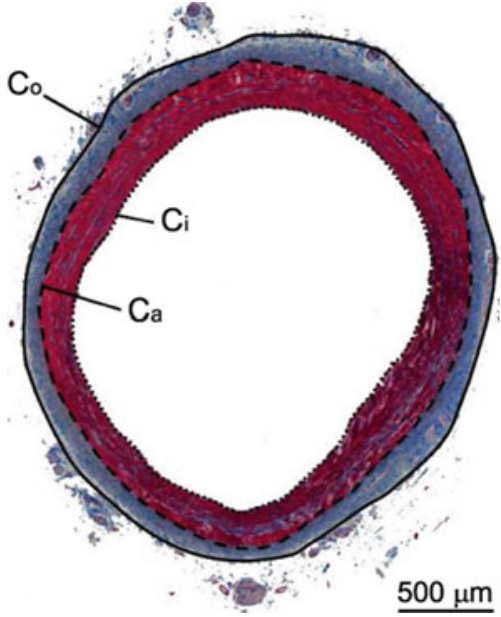

Fig. 4 Histological section of an LAD stained with MTC with the inner circumference $\left(C_{i}\right.$, dotted line $)$, inner circumference of the adventitia layer $\left(C_{a}\right.$, dashed line $)$, and the outer circumference $\left(C_{o}\right.$, solid line $)$ used to determine geometric parameters. Muscular and connective tissue are colored pink and collagen is stained blue

Table 1 Geometric parameter values of LAD 1-7 and their means and standard deviations (SD); $R_{i}$ and $H_{0}$ the final estimate of the unloaded inner radius and wall thickness from the fit procedure; $A_{0}$ the crosssectional area of the unloaded artery; $\kappa=h / r_{i}$ at $P \approx 13.3 \mathrm{kPa}$ and $\lambda_{z, \text { phys }} ; \phi_{f}$ the collagen fiber fraction; $\lambda_{z \text {,phys }}$ the physiological axial pre-stretch

\begin{tabular}{lllllll}
\hline LAD & $R_{i}(\mathrm{~mm})$ & $H_{0}(\mathrm{~mm})$ & $A_{0}\left(\mathrm{~mm}^{2}\right)$ & $\kappa$ & $\phi_{f}$ & $\lambda_{z, \text { phys }}$ \\
\hline 1 & 1.25 & 0.34 & 3.02 & 0.092 & 0.37 & 1.36 \\
2 & 1.52 & 0.39 & 4.23 & 0.088 & 0.39 & 1.49 \\
3 & 1.14 & 0.29 & 2.33 & 0.086 & 0.35 & 1.39 \\
4 & 1.23 & 0.33 & 2.89 & 0.113 & 0.45 & 1.41 \\
5 & 1.36 & 0.31 & 2.94 & 0.105 & 0.48 & 1.34 \\
6 & 1.07 & 0.29 & 2.25 & 0.064 & 0.41 & 1.38 \\
7 & 1.32 & 0.32 & 2.96 & 0.112 & 0.42 & 1.36 \\
Mean & 1.27 & 0.32 & 2.95 & 0.094 & 0.41 & 1.39 \\
SD & 0.15 & 0.04 & 0.65 & 0.017 & 0.05 & 0.05 \\
\hline
\end{tabular}

the wall thickness and the inner radius at physiological loading was $0.09 \pm 0.02$.

The experimentally measured $P-\Delta F_{z}$ and $P-r_{i}$ relations, the model fits, and the resulting parameter sets are shown in Figs. $5 \mathrm{~b}-\mathrm{h}, 6 \mathrm{~b}-\mathrm{h}$, and 7 , respectively. The variation in the fitted parameters of the seven sets is larger for $k_{1}$ and $k_{2}$ than for $G$ and $\beta$ (Fig. 7). The optimal parameter sets $\Upsilon_{j}$ resulted in a good quality of the fits of the $P-r_{i}$ relations with average relative deviations of $0.005 \pm 0.003\left(\delta_{r}\right)$, corresponding to a deviation of $8.6 \mu \mathrm{m}$ on average (Table 3). The optimal model approximations of the $P-\Delta F_{z}$ relations resulted in an average relative deviation of the maxima and minima from the $\Delta F_{z}$ range of $0.2 \pm 0.1\left(\delta_{F}\right)$, corresponding to an average deviation of about $4 \mathrm{mN}$ from the experimentally measured axial force range. Averaging the sets $\Upsilon_{1-7}$ resulted in $\bar{\Upsilon}$ (Table 2), and fitting the average data of LAD 1-7 (Figs. 5a, 6a) resulted in the generic set $\Upsilon_{m}$ (Table 2). The parameters $G$ and $k_{1}$ of set $\bar{\Upsilon}$ are smaller than the corresponding parameters of set $\Upsilon_{m}$, whereas $k_{2}$ and $\beta$ of the two sets correspond well (difference $<10 \%$ ). Using the generic parameter sets in combination with the generic geometric parameters (Table 2) with a given LAD-specific $r_{i}$ at physiological loading to approximate the $P-\Delta F_{z}$ and the $P-r_{i}$ relations, resulted in the relations as shown in Figs. $5 \mathrm{~b}-\mathrm{h}$ and $6 \mathrm{~b}-\mathrm{h}$. The deviations of the model approximations from the experimental data increased when a generic parameter set was used compared to the use of $\Upsilon_{j} . \delta_{F}$ increased more when $\bar{\Upsilon}$ was used than with $\Upsilon_{m}(3 \times$ vs. $1.5 \times$ increase, Table 3$)$ and the increase was similar for $\delta_{r}(4.5 \times$, Table 3$)$.

\section{Discussion}

In this study a generic description of the passive mechanical behavior of the porcine LAD is presented by prescribing generic geometric and material parameters to be used with the model by Driessen et al. (2005). Contrary to other studies, the generic parameters that were obtained have been applied to each tested artery using a single radius measurement at physiological loading to obtain artery-specific $P-r_{i}$ and $P-\Delta F_{z}$ relations, which compared well to the relations measured in vitro.

The experimental method, with maximum pressures still physiological and measurements at physiological stretch, differs from other studies. For e.g., Schulze-Bauer et al. (2003) loaded human iliac arteries with a maximum pressure of $250 \mathrm{mmHg}$ and, in contrast to our study, an axial force $(0-9.9 \mathrm{~N})$ while measuring the resulting axial stretch. Holzapfel et al. (2005) performed uniaxial tests to determine layer-specific mechanical properties of human coronary arteries. We preferred measurements to be performed in a physiological environment while maintaining the artery's structural integrity. Accordingly, we let the maximum load not exceed maximal physiological levels to prevent arterial wall damage and used whole artery segments. Maintaining the tubular shape of the arterial segments, nevertheless, had the disadvantage that layer-specific material parameters could not be obtained, as layers could not be separated while maintaining segments free of any leakage.

In this study, only the axial force change during a pressure cycle (i.e. $\Delta F_{z}$ ) has been considered due to a possible inaccuracy in the absolute axial force measurements. Moreover, it has been shown that when the $P-r_{i}$ and $P-F_{z}$ relations are fitted simultaneously using a fiber-reinforced model as used in this study, the results are relatively poor (Zulliger et al. 2004; Rezakhaniha and Stergiopulos 2008). Hence, Rezakhaniha and Stergiopulos (2008) included an anisotropic 

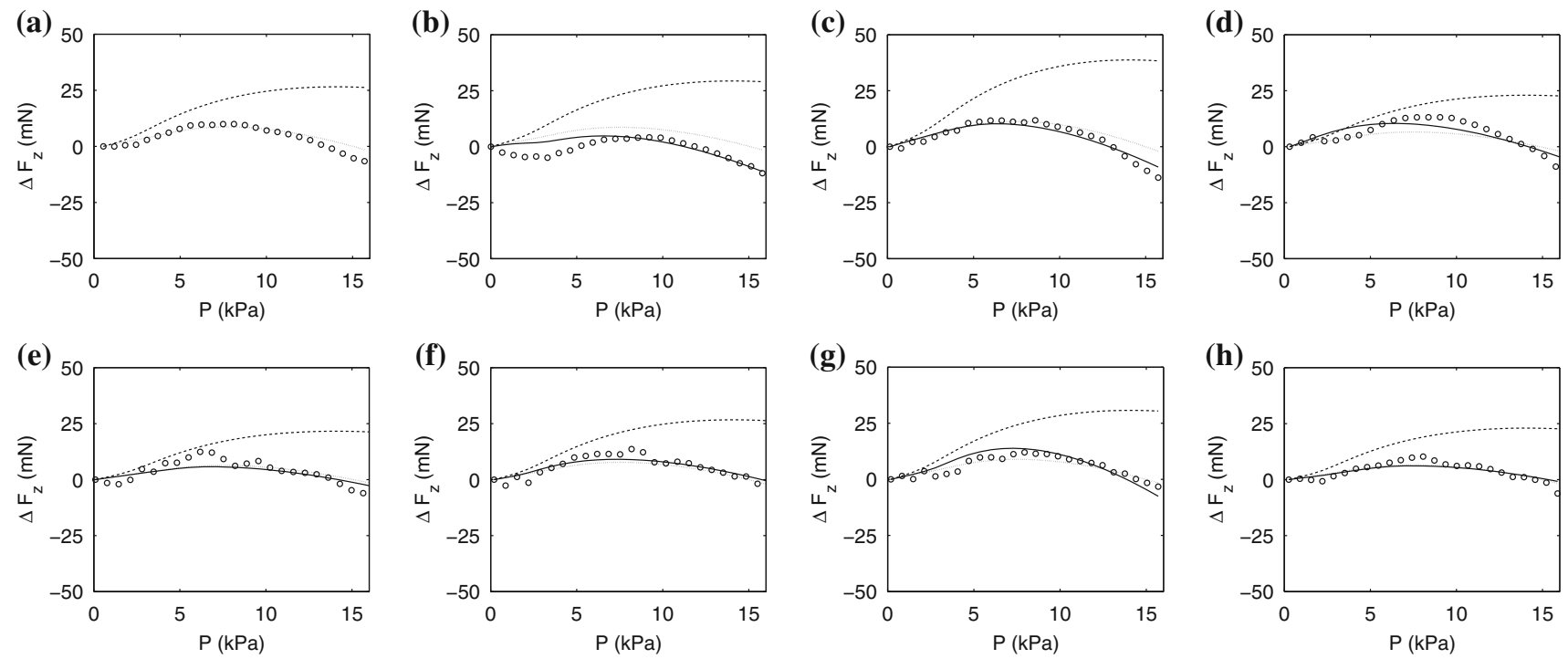

Fig. 5 Model approximations of the experimental $P-\Delta F_{z}$ data (circles) of the mean data of LAD 1-7 (a) and of LAD 1-7 (b-h) using $\Upsilon_{i}$ (solid lines), $\bar{\Upsilon}$ (dashed lines), and $\Upsilon_{m}$ (dotted lines)
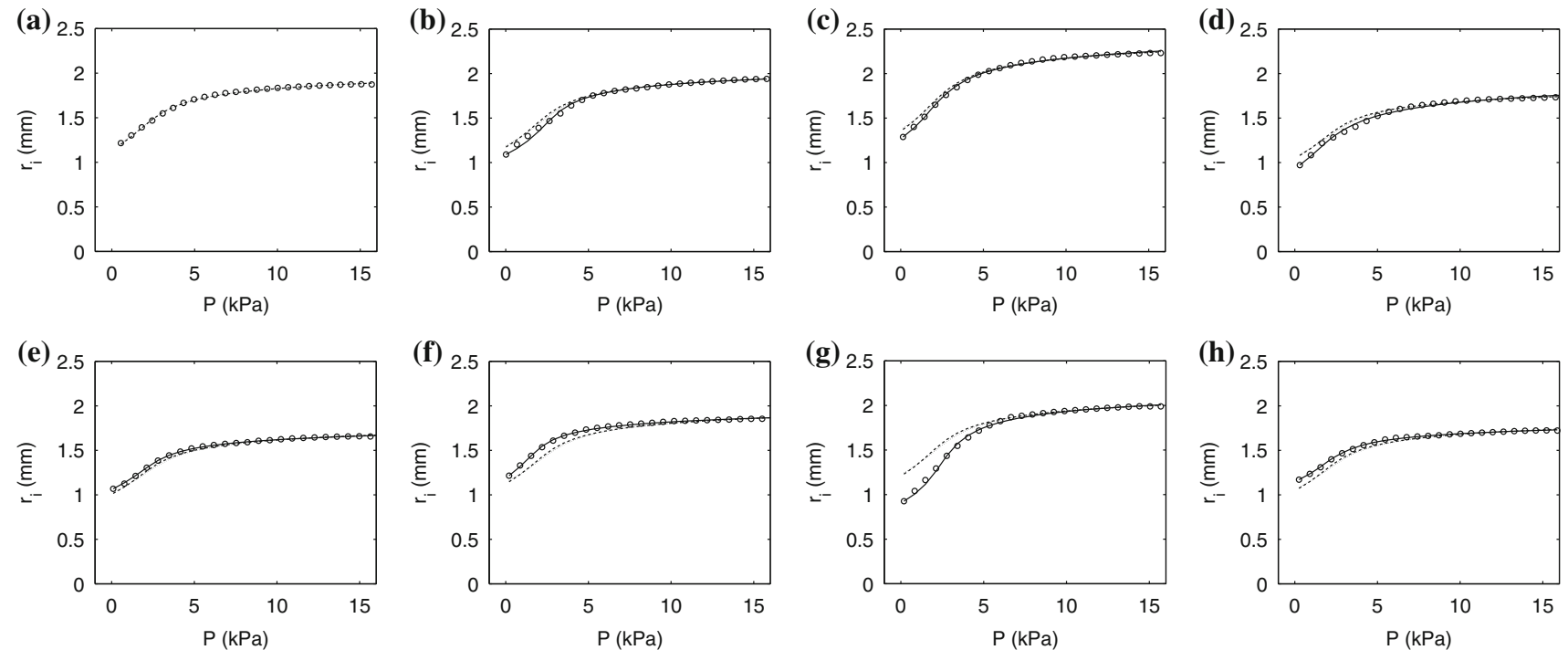

Fig. 6 Model approximations of the experimental $P-r_{i}$ data (circles) of the mean data of LAD 1-7 (a) and of LAD 1-7 (b-h) using $\Upsilon_{i}$ (solid lines), $\bar{\Upsilon}$ (dashed lines), and $\Upsilon_{m}$ (dotted lines)

elastin structure in the model to overcome this problem. In future studies, it would therefore be interesting to fit the $P-F_{z}$, instead of $P-\Delta F_{z}$ behavior while considering the findings of the study mentioned above. It should be noted, though, that when elastin anisotropy is taken into account this also increases the number of material parameters.

Residual strains were not accounted for in this study. As the stress distribution through the vessel wall is of interest, including the residual strain might be of interest as well. Since measuring the opening angle as a measure of residual strain leads to very heterogenous results, a better option would be to include the opening angle as an extra fit parameter using a method as presented by e.g. van Dyke and Hoger (2002). However, we tried to keep the number of fit parameters to a minimum, and therefore, it was decided not to include the residual strain in this study.

The generic geometrical parameters $\bar{\phi}_{f}, \bar{\kappa}$, and $\bar{\lambda}_{z \text {,phys }}$ were derived by averaging the measurements of the seven tested LADs (Tables 1, 2). The collagen fiber volume fraction $\bar{\phi}_{f}$ was derived from histological data by determination of the relative thickness of the adventitia, as collagen is mainly located in this layer (Rhodin 1980). Although the adventitia not only consists of collagen, it was assumed that this was compensated by neglecting the collagen in other parts of the 


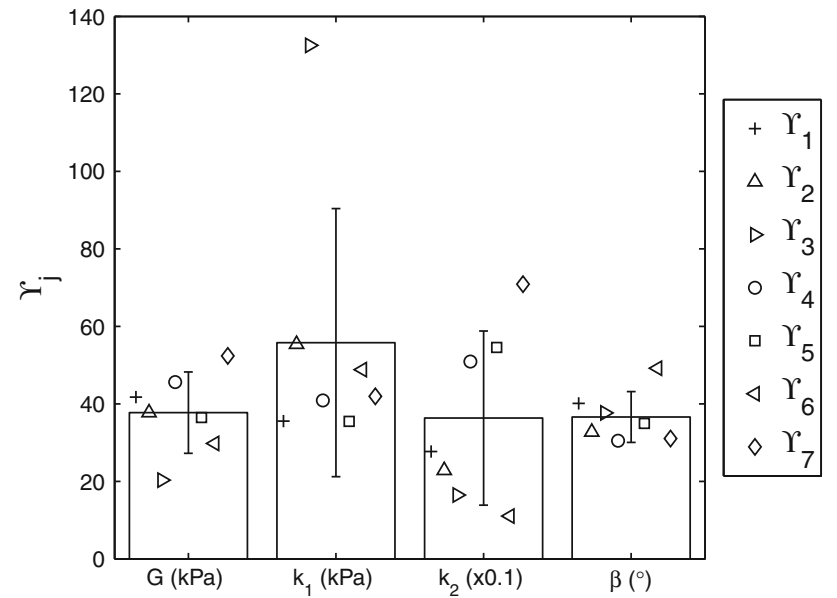

Fig. 7 The parameter values of the parameter sets $\Upsilon_{j}(j=1, \ldots, 7)$. Bars and errorbars represent the mean values \pm SD of the parameters of $\Upsilon_{1-7}$

Table 2 Parameter values of the generic sets $\bar{\Upsilon}$ and $\Upsilon_{m}$, and the generic geometric parameters used as a generic input for the constitutive model

\begin{tabular}{lccc}
\hline & $\bar{\Upsilon}$ & $\Upsilon_{m}$ \\
\hline $\mathrm{G}(\mathrm{kPa})$ & 38 & & 46 \\
$k_{1}(\mathrm{kPa})$ & 56 & & 64 \\
$k_{2}(-)$ & 3.6 & & 3.6 \\
$\beta\left({ }^{\circ}\right)$ & 37 & & 34 \\
$\bar{\lambda}_{z, \text { phys }}$ & & 1.39 & \\
$\bar{\phi}_{f}$ & & 0.41 & \\
$\bar{\kappa}$ & & 0.09 & \\
\hline
\end{tabular}

Table 3 Mean relative deviations \pm SD of the model approximations from the experimental data of LAD 1-7 using $\Upsilon_{j}, \bar{\Upsilon}$, and $\Upsilon_{m}$

\begin{tabular}{lll}
\hline & $\bar{\delta}_{F}$ & $\bar{\delta}_{r}$ \\
\hline$\Upsilon_{j}$ & $0.20 \pm 0.10$ & $0.005 \pm 0.003$ \\
$\bar{\Upsilon}$ & $0.64 \pm 0.24$ & $0.023 \pm 0.015$ \\
$\Upsilon_{m}$ & $0.29 \pm 0.12$ & $0.023 \pm 0.014$ \\
\hline
\end{tabular}

arterial wall. The volume fraction of 0.41 found in this study is in the same range as the 0.48 fraction of collagen per dry weight found by Fischer and Llaurado (1966) for the canine coronary artery. A possible under- or overestimation of $\bar{\phi}_{f}$ used in this study will mostly be compensated by a higher or lower $k_{1}$ as those two are directly related in the fiber part of the constitutive model. The wall thickness to inner radius ratio at $13.3 \mathrm{kPa}$ was 0.09 with a small standard deviation of 0.02 , which would be expected as circumferential stress levels are assumed to be comparable for different arteries. This ratio corresponds to the value of 0.10 for porcine coronary arteries derived from the data by Lu et al. (2004).

All $P-r_{i}$ and $P-\Delta F_{z}$ measurements were performed at the stretch at which the axial force is pressure-invariant (referred to as $\lambda_{z \text {,phys }}$ ). A clear transition stretch at which the axial force range during a pressure cycle was minimal could be observed. On average, $\lambda_{z}$,phys was found to be $1.39 \pm 0.05$, which is the same as the in vivo stretch for the canine coronary artery in Humphrey et al. (2009). The typical $P-F_{z}$ behavior at in situ stretch has been shown for rat carotid arteries (Weizsäcker et al. 1983) and dog carotid, femoral, and aortic arteries (van Loon 1977). To the best of our knowledge, this has not yet been proven for porcine coronary arteries. Kassab et al. (2002) did derive a measure of the physiological stretch by pressurizing the main coronary arteries in an isolated heart preparation at $80 \mathrm{mmHg}\left(\lambda_{z}=1.32 \pm 0.11, n=5\right)$. An underestimation of the physiological stretch of the LAD in that study would be expected as pressures in the unloaded heart were below physiological levels. Nevertheless, we compared the derived mean $\lambda_{z \text {,phys }}$ from our study to the stretch found in that study and no significant difference in the stretches was found (P-value of 0.24$)$. It would be preferred to validate this stretch in a more accurate way. Measuring the in vivo stretch of the coronary arteries is rather complicated as they are located on a beating heart and in the ex vivo situation, as beating has ceased, pressure load on the heart, and thus stretch, are reduced. In spite of the complexity of such a measurement, information on the in vivo stretch of the coronary artery is desired.

The experimentally measured reduced axial force range at $\lambda_{z \text {,phys }}$ was $20 \mathrm{mN}$ on average (Fig. 5), compared to an absolute axial force of $150-200 \mathrm{mN}$. The $P-r_{i}$ relations show the typical arterial stiffening behavior (Fig. 6), which is captured by the exponential relation for the fiber stress in the model (Eq. 3). An assumption was made for the distribution of the fiber orientation, using a periodic approximation of the normal probability distribution with a dispersion of $10^{\circ}$ (Eq. 6). Using a technique that enables visualization of collagen, like confocal microscopy, would make it possible to use a possibly more physiological distribution in future studies.

The relations of each artery were fitted (Figs. 5, 6) resulting in the optimal fit parameter sets $\Upsilon_{j}$ (Fig. 7). There is quite some spread in the parameter values between the different individual data sets, with the highest standard deviations on the fiber parameters $k_{1}$ and $k_{2}$ (Fig. 7). The $P-r_{i}$ relation of LAD 3 does not reveal a clear s-shape, resulting in a low shear modulus and high $k_{1}$. LAD 4, 5, and 7 show arterial stiffening at a lower pressure, correlating with the higher value found for the fit parameter $k_{2}$. LAD 6 shows the largest change in $r_{i}$, resulting in a lower value of the fitted shear modulus and the fiber parameters, and a higher mean fiber orientation $\beta$ corresponding to a more axially oriented arrangement of 
the fibers. The fits correspond well with the experimentally measured relations for the whole pressure range. The resulting average deviation from the maxima and minima of the $P-\Delta F_{z}$ relations was about $4 \mathrm{mN}\left(\bar{\delta}_{F}=0.20\right)$, and the average deviation from the $P-r_{i}$ relations was $8.5 \mu \mathrm{m}\left(\bar{\delta}_{r}=0.005\right)$. In addition, preliminary studies have shown that the modeled distribution on the fiber orientation led to a better fit of the experimental data than when this distribution was not accounted for. From this it can be concluded that the constitutive model of Driessen et al. (2005) that was used in this study is well capable of describing the $P-\Delta F_{z}$ and $P-r_{i}$ relations of the passive porcine coronary artery.

The generic sets $\bar{\Upsilon}$ and $\Upsilon_{m}$ correspond well for $k_{2}$ and $\beta$, whereas the parameters $G$ and $k_{1}$ are higher for the set $\Upsilon_{m}$, indicating stiffer arterial wall behavior (Table 2). The difference in the generic parameter sets can be attributed to the non-linearity of the constitutive model. The model approximations of the $P-\Delta F_{z}$ relations deviate less from the experimental data when $\Upsilon_{m}$ instead of $\bar{\Upsilon}$ was used. This can be ascribed to the fact that the set $\Upsilon_{m}$ was derived from the fit of the averaged mechanical relations of all seven arteries at the

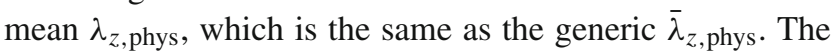
averaged $P-\Delta F_{z}$ relation shows a small axial force range. The parameters of $\Upsilon_{m}$ describe this small axial force range at $\bar{\lambda}_{z, \text { phys }}$, which was used as a generic input. As the axial force range of the individual experimental data sets was small as well, deviations are smaller when $\Upsilon_{m}$ was used.

The $P-r_{i}$ approximation using a generic set was made LAD-specific by introducing the measured $r_{i}$ at physiological loading as an LAD-specific input. This $r_{i \text {,phys }}$ was approximated iteratively with the model using the generic parameters. Consequently, the model approximates the experimentally measured $P-r_{i}$ relation at physiological pressures very well (Fig. 6). At lower pressures more deviation was found, especially for LAD 6 with $\Upsilon_{6}$, which has parameter values different from the other parameter sets $\Upsilon_{j}$. Using the generic parameter sets $\bar{\Upsilon}$ and $\Upsilon_{m}$ resulted in an increase of $\bar{\delta}_{F}$ to 0.64 and 0.29 , respectively, the latter corresponding to an average deviation from the experimentally measured axial force range of only $6 \mathrm{mN}$. The approximation of the $P-r_{i}$ relations resulted in a relative deviation of 0.023 for both generic sets, corresponding to a deviation of only $32 \mu \mathrm{m}$ on average, which is in the same order as the measuring accuracy of the change in diameter of the used ultrasound machine $(=31 \mu \mathrm{m})$.

Summarizing, a generic geometric parameter set in combination with two generic model parameter sets for the model by Driessen et al. (2005) describing the passive mechanical behavior of the porcine LAD has been proposed. The generic model parameter set $\Upsilon_{m}$ is preferred as using this set results in a more accurate approximation of the $P-\Delta F_{z}$ relation. Using a single radius measurement at physiological loading, the $P-\Delta F_{z}$ and $P-r_{i}$ relations, as a measure of the mechanical behavior of the porcine LAD, can be predicted with an accuracy of about $6 \mathrm{mN}$ ( $=29 \%$ relative to the $\Delta F_{z}$ range) and $32 \mu \mathrm{m}(=2.3 \%)$ on average. The proposed model in combination with the generic parameters of $\Upsilon_{m}$ (Table 2) enables prediction of the behavior of a specific porcine coronary artery for a larger pressure range by performing a single radius measurement at physiological loading.

Open Access This article is distributed under the terms of the Creative Commons Attribution Noncommercial License which permits any noncommercial use, distribution, and reproduction in any medium, provided the original author(s) and source are credited.

\section{References}

Chuong CJ, Fung YC (1984) Compressibility and constitutive equation of arterial wall in radial compression experiments. J Biomech 17(1):35-40

Cox RH (1983) Comparison of arterial wall mechanics using ring and cylindrical segments. Am J Physiol 244(2):H298-H303

Driessen NJ, Bouten CV, Baaijens FP (2005) A structural constitutive model for collagenous cardiovascular tissues incorporating the angular fiber distribution. J Biomech Eng 127(3):494-503

Driessen NJ, Cox MA, Bouten CV, Baaijens FP (2008) Remodelling of the angular collagen fiber distribution in cardiovascular tissues. Biomech Model Mechanobiol 7(2):93-103

Driessen NJ, Wilson W, Bouten CV, Baaijens FP (2004) A computational model for collagen fibre remodelling in the arterial wall. J Theor Biol 226(1):53-64

Fischer GM, Llaurado JG (1966) Collagen and elastin content in canine arteries selected from functionally different vascular beds. Circ Res 19(2):394-399

Hayashi K (1993) Experimental approaches on measuring the mechanical properties and constitutive laws of arterial walls. J Biomech Eng 115(4B):481-488

Holzapfel GA, Gasser TC (2000) A new constitutive framework for arterial wall mechanics and a comparative study of material models. J Elasticity 61:1-48

Holzapfel GA, Sommer G, Gasser CT, Regitnig P (2005) Determination of layer-specific mechanical properties of human coronary arteries with nonatherosclerotic intimal thickening and related constitutive modeling. Am. J. Physiol Heart Circ. Physiol 289(5):H2048-H2058

Humphrey JD, Eberth JF, Dye WW, Gleason RL (2009) Fundamental role of axial stress in compensatory adaptations by arteries. J Biomech 42(1):1-8

Humphrey JD, Na S (2002) Elastodynamics and arterial wall stress. Ann Biomed Eng 30(4):509-523

Kassab GS, Gregersen H, Nielsen SL, Lu X, Tanko LB, Falk E (2002) Remodelling of the left anterior descending artery in a porcine model of supravalvular aortic stenosis. J Hypertens 20(12):2429-2437

Lally C, Reid AJ, Prendergast PJ (2004) Elastic behavior of porcine coronary artery tissue under uniaxial and equibiaxial tension. Ann Biomed Eng 32(10):1355-1364

Lu X, Pandit A, Kassab GS (2004) Biaxial incremental homeostatic elastic moduli of coronary artery: two-layer model. Am J Physiol Heart Circ Physiol 287(4):H1663-H1669

Rezakhaniha R, Stergiopulos N (2008) A structural model of the venous wall considering elastin anisotropy. J Biomech Eng 130(3):031017 
Rhodin JAG (1980) Handbook of physiology: the cardiovascular system. architecture of the vessel wall. Book chapter 1. American Physiology Society, USA 1-31

Schulze-Bauer CA, Morth C, Holzapfel GA (2003) Passive biaxial mechanical response of aged human iliac arteries. J Biomech Eng 125(3):395-406

van Dyke TJ, Hoger A (2002) A new method for predicting the opening angle for soft tissues. J Biomech Eng 124(4):347-354

van Loon P (1977) Length-force and volume-pressure relationships of arteries. Biorheology 14(4):181-201
Weizsäcker HW, Lambert H, Pascale K (1983) Analysis of the passive mechanical properties of rat carotid arteries. J Biomech 16(9):703-715

Zulliger MA, Fridez P, Hayashi K, Stergiopulos N (2004) A strain energy function for arteries accounting for wall composition and structure. J Biomech 37(7):989-1000 